\title{
Caracterización de la capa y el filo adherido en el mecanizado de la aleación AA7050
}

\author{
J.M. Sánchez-Sola", M.A. Sebastián", M.S. Carrilero", J.M. González* y M. Marcos"
}

Resumen El filo recrecido (BUE) y capa adherida (BUL) son los principales efectos de incorporación de material mecanizado a la herramienta de corte. Ambos, se enmarcan dentro de los denominados efectos de adhesión. La existencia de BUL y BUE puede influir de forma directa sobre la calidad de acabado superficial de la pieza e indirectamente sobre el desgaste de la propia herramienta. En el presente trabajo se analizan las diferencias microestructurales entre el BUL y el BUE, formados en el mecanizado de la aleación AA7050. Estas diferencias ponen de manifiesto que, al menos en las aleaciones estudiadas, ambos fenómenos están gobernados por distintos mecanismos y, por tanto, no pueden considerarse como formas diferentes de un mismo efecto.

Palabras clave Filo recrecido. BUE. Capa adherida. BUL. Aleación AA7050.

\section{Characterization of the built-up edge and the built-up layer in the machining process of AA7050 alloy}

\begin{abstract}
The built-up edge (BUE) and the built-up layer (BUL) are the most common adhesion effects that can take place in the metal machining processes. Surface finish quality and tool life can be negatively affected by BUL and BUE. This work reports the results of a study on the microstructural differences between BUL and BUE formed in AA7050 cutting processes. From this study, it can be concluded that different mechanisms are the responsible of BUL and BUE. Thus, BUL and BUE cannot be considered as different forms of the same effect.
\end{abstract}

Keywords

Built-up edge. BUE. Built-up layer. BUL. AA7050 alloy.

\section{INTRODUCCIÓN}

El diseño de un proceso de mecanizado se encuentra sujeto a objetivos que se plantean sobre la base de la relación economía-calidad. Así, por una parte, las piezas obtenidas deben responder a unos criterios de acabado superficial prefijados y haber sido fabricadas dentro de los límites económicos disponibles ${ }^{[1]}$. La obtención de esa calidad superficial en las piezas mecanizadas está ligada, entre otros factores, al mantenimiento de la geometría y propiedades iniciales de la herramienta seleccionada para llevar a cabo el proceso de corte ${ }^{[1 \text { y } 2]}$. Así, el tiempo de la estabilidad geométrica, dentro del rango establecido por el criterio de desgaste aceptado, es una medida de la vida de la herramienta ${ }^{[2}$ y 3$]$.
Por otro lado, debe tenerse en cuenta que, durante el desarrollo de un proceso de mecanizado por arranque de viruta, la herramienta se encuentra sometida a la acción combinada de grandes tensiones mecánicas, las cuales pueden ser suficientes para alterar la geometría de la herramienta. Adicionalmente, en el proceso de corte se liberan, localmente, altas cantidades de energía térmica, que pueden provocar elevados aumentos locales de la temperatura, suficientes para modificar las condiciones mecánicas de la herramienta y, por ende, su estado inicial. Otras causas, como la abrasión por los impactos a alta velocidad de partículas separadas del material de la pieza sobre la cara de desprendimiento de la herramienta o la acción corrosiva de lubricantes y refrigerantes pueden, asimismo, causar el desgaste de

$\left({ }^{*}\right)$ Departamento de Ingeniería de Construcción y Fabricación, UNED, Escuela Técnica Superior de Ingenieros Industriales. Apdo. 60249, E-28080, Madrid, SPAIN.

$\left.{ }^{(* *}\right)$ Departamento de Ingeniería Mecánica y Diseño Industrial, Universidad de Cádiz, Escuela Superior de Ingeniería. c/Chile s/n, E-11003, Cádiz, SPAIN. Tel. +34 956.015123. Fax.+34 956.015101.E-mail: mariano.marcos@uca.es 
la herramienta. Además, todos estos factores no intervienen de forma aislada sino que su acción combinada suele producir un efecto sinérgico que aumenta la pérdida de las condiciones ideales de la geometría de la herramienta.

De acuerdo con los comentarios anteriores, el desgaste de la herramienta de corte responde a un mecanismo complejo, lo que hace casi imposible abordar su estudio de forma general. Sin embargo, en determinadas condiciones, la contribución de algún mecanismo concreto es muy superior a la de los restantes, pudiendo considerarlo como el único responsable del deterioro de la herramienta.

El mecanismo de adhesión es uno de los principales responsables del desgaste de herramientas de corte, presentándose en un amplio rango de temperaturas. Mediante este mecanismo, partículas de la herramienta quedan adheridas a la viruta o a la pieza mecanizada ${ }^{[4]}$.

Sin embargo, este proceso de adhesión puede darse, también, en sentido contrario mediante la incorporación de fragmentos de material de la pieza a la superficie de la herramienta. Generalmente, estos fragmentos son mecánicamente inestables, pudiendo arrancar partículas de la herramienta provocando su desgaste. El material de la pieza adherido a la superficie de desprendimiento de la herramienta puede localizarse en el filo, dando lugar a la formación del filo recrecido (Built-up edge, B.U.E.) o extenderse a zonas más amplias de la cara de desprendimiento de la herramienta, originando la llamada capa adherida (Built-up layer, B.U.L.) ${ }^{[5 \mathrm{y} 6]}$.

Originalmente, tanto el BUL como el BUE fueron considerados como distintas formas de un mismo efecto ${ }^{[4]}$.

Más recientemente, a partir de consideraciones sobre la distribución de temperaturas en la herramienta, se han establecido diferencias entre los mecanismos de formación de ambos efectos para el torneado de aleaciones de $\mathrm{Al}-\mathrm{Cu}{ }^{[5]}$.

En este trabajo se lleva a cabo un estudio mediante SEM y EDS sobre las diferencias en el mecanismo de formación de BUL y BUE en distintos procesos de mecanizado de la aleación AA7050 (Al-Zn).

\section{MATERIALES Y PROCEDIMIENTO EXPERIMEN- TAL}

Como material de estudio se empleó la aleación AA 7050(Al-Zn), cuya composición, determinada mediante ICP, se incluye en la tabla I.
Tabla I. Composición de la aleación AA7050 (\% en masa) Table I. Composition (\% mass) of AA7050 alloy

\begin{tabular}{ccccccccc}
\hline $\mathrm{Cu}$ & $\mathrm{Mg}$ & $\mathrm{Mn}$ & $\mathrm{Si}$ & $\mathrm{Fe}$ & $\mathrm{Zn}$ & $\mathrm{Ti}$ & $\mathrm{Cr}$ & $\mathrm{Al}$ \\
\hline 2,30 & 2,3 & 0,10 & 0,12 & 0,15 & 6,2 & 0,06 & 0,04 & Resto \\
\hline
\end{tabular}

Se efectuaron ensayos de cilindrado en un torno horizontal EMCOTURN-242 T, equipado con Control Numérico EMCOTRONIC TM02, empleando velocidades de corte no superiores a los $200 \mathrm{~m} / \mathrm{min}$, con objeto de garantizar la formación de filo recrecido y avances comprendidos entre 0,05 y $0,3 \mathrm{~mm} / \mathrm{rev}$. La profundidad de corte se fijó a un valor de $2 \mathrm{~mm}$. La duración de los ensayos no excedió de los $10 \mathrm{~s}$. En este caso, las muestras trabajadas fueron barras cilíndricas con longitud comprendida entre 300 y $400 \mathrm{~mm}$ y diámetro entre 80 y $120 \mathrm{~mm}$. Las herramientas empleadas fueron plaquitas de TiN con denominación ISO KCMW 11 T3 08 FN M-.

De igual forma, se llevaron a cabo ensayos de taladrado en un Centro de Mecanizado EMCO VM-300 en condiciones de mecanizado análogas a las descritas para los procesos de torneado, empleando brocas de TiN y WC-Co.

El proceso de corte fue monitorizado haciendo uso de una Cámara Digital NIKON COOLPIX 4500. Al finalizar los ensayos, las herramientas empleadas fueron observadas con un Microscopio Electrónico de Barrido JEOL 820-SM. El material adherido a la superficie de la herramienta fue analizado mediante Espectroscopía de Energía Dispersiva con un analizador LINK, AN-10000, acoplado al citado microscopio.

\section{RESULTADOS Y DISCUSIÓN}

En la figura 1 se incluye la imagen SEM correspondiente a una herramienta de $\mathrm{TiN}$, tras un proceso de $10 \mathrm{~s}$ de torneado de la aleación AA7050 a 170 $\mathrm{m} / \mathrm{min}$ con un avance de $0,1 \mathrm{~mm} / \mathrm{rev}$. En dicha figura, se pueden distinguir dos zonas con formas diferentes en las que se aprecia una alteración de la geometría de la herramienta. En efecto, en la zona más próxima al filo puede observarse una acumulación de material con mayor espesor (BUE) que el que se extiende sobre parte de la cara de desprendimiento (BUL) con un espesor menor. La formación de BUL y BUE fue detectada para otros materiales de herramienta y otros procesos de mecanizado, como el taladrado (Fig. 2). 


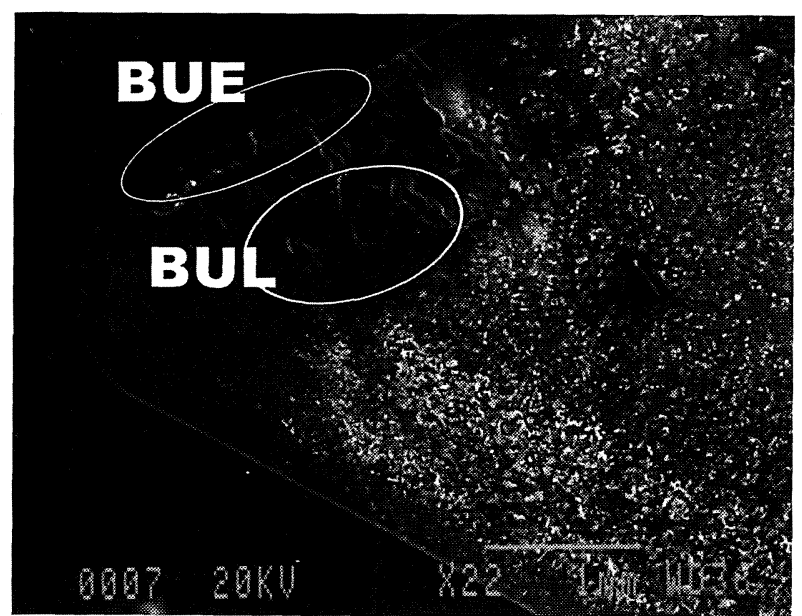

Figura 1. Imagen SEM adquirida sobre una plaquita con recubrimiento TiN, tras $10 \mathrm{~s}$ de tornado horizontal de la aleación AA7050.

Figure 1. SEM image acquired on a TiN insert after $10 \mathrm{~s}$ of horizontal turning of AA7050 alloy.

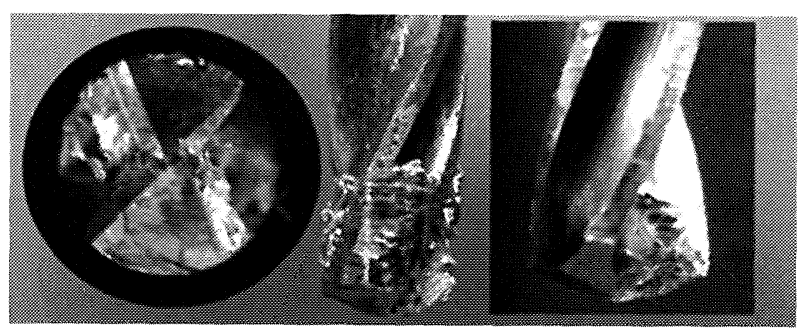

Figura 2. Incorporación de material mecanizado a brocas de WC-Co y HSS, tras diferentes tiempos de taladrado de placas de la aleación AA7050.

Figure 2. Adhesion of drilled material to WC-Co and HSS tools after different times of drilling AA7050 samples.

La morfología del BUL y el BUE es aparentemente similar y la diferencia inicial parece estar relacionada, única y exclusivamente, con el espesor. Sin embargo, el análisis mediante EDS de ambos efectos pone de manifiesto la existencia de, al menos, notables diferencias en la composición de ambos. En la figura 3 se han superpuesto los espectros EDS adquiridos, a la misma escala, en zonas correspondientes al BUL y al BUE. Como referencia se incluye el espectro adquirido sobre una muestra de la aleación AA7050 antes de ser mecanizada.

Como puede apreciarse en la figura 3 , los picos asociados a los elementos aleantes en el BUE presentan una intensidad relativa muy semejante a los correspondientes a la aleación AA7050. Sin embargo, en el caso de la capa adherida, parece existir un notable descenso en estos picos de intensidad, aproximándose al espectro correspondiente al aluminio puro.

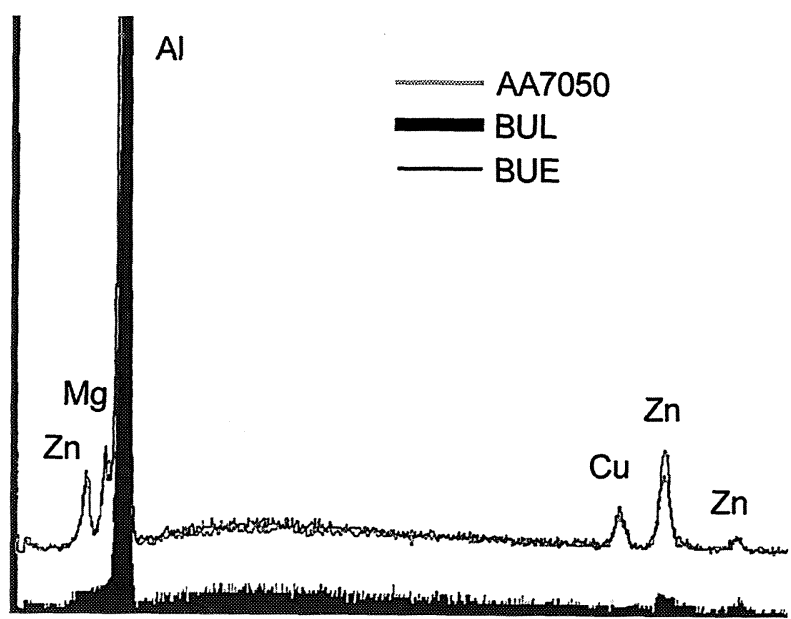

Figura 3. Comparación de los espectros EDS del BUL, el BUE y la aleación.

Figure 3. Comparison of EDS spectra corresponding to BUL, $B U E$ and $A A 7050$ alloy.

Para poder explicar estas diferencias se han analizado, por una parte, los efectos de adhesión sobre herramientas para mecanizados de duración inferiores a $1 \mathrm{~s}$. En la figura 4 se incluyen imágenes SEM correspondientes a herramientas empleadas en procesos de torneado de 0,5 (a) y 0,1 (b) s de duración. Como puede apreciarse en esta figura no se observa la formación de filo recrecido, desarrollándose sólo la formación de una capa metálica correspondiente al BUL, cuyo espectro EDS puede identificarse con el del aluminio de alta pureza. De acuerdo con estos resultados, el BUL y el BUE no son efectos que se produzcan simultáneamente, sino que el filo adherido se forma una vez desarrollado el BUL y, además, tienen composiciones muy diferentes.

Por otro lado, el análisis de la aleación ha mostrado la presencia de tres tipos de partículas intermetálicas en la misma: partículas esféricas enriquecidas en cobre y magnesio $\left(\mathrm{Al}_{2} \mathrm{CuMg}\right)$; partí- culas alargadas e irregulares $\left(\mathrm{Al}_{7} \mathrm{Cu}_{2} \mathrm{Fe}\right)$ y partículas globulares $\left(\mathrm{Mg}_{2} \mathrm{Si}\right)^{[7]}$. El punto de fusión de estas partículas es mayor que el correspondiente al aluminio puro. A partir de esta consideración, y teniendo en cuenta la existencia de temperaturas del orden de los $700^{\circ} \mathrm{C}$ en los procesos de mecanizado empleados ${ }^{[6]}$, no puede pensarse en un proceso inicial de formación del BUL en el que sólo intervengan fuerzas de adhesión. En efecto, el mecanismo de formación de la capa adherida puede suponerse sobre la base de la incipiente fusión inicial de la matriz de aluminio, que se suelda sobre la cara de desprendimiento de la herramienta 

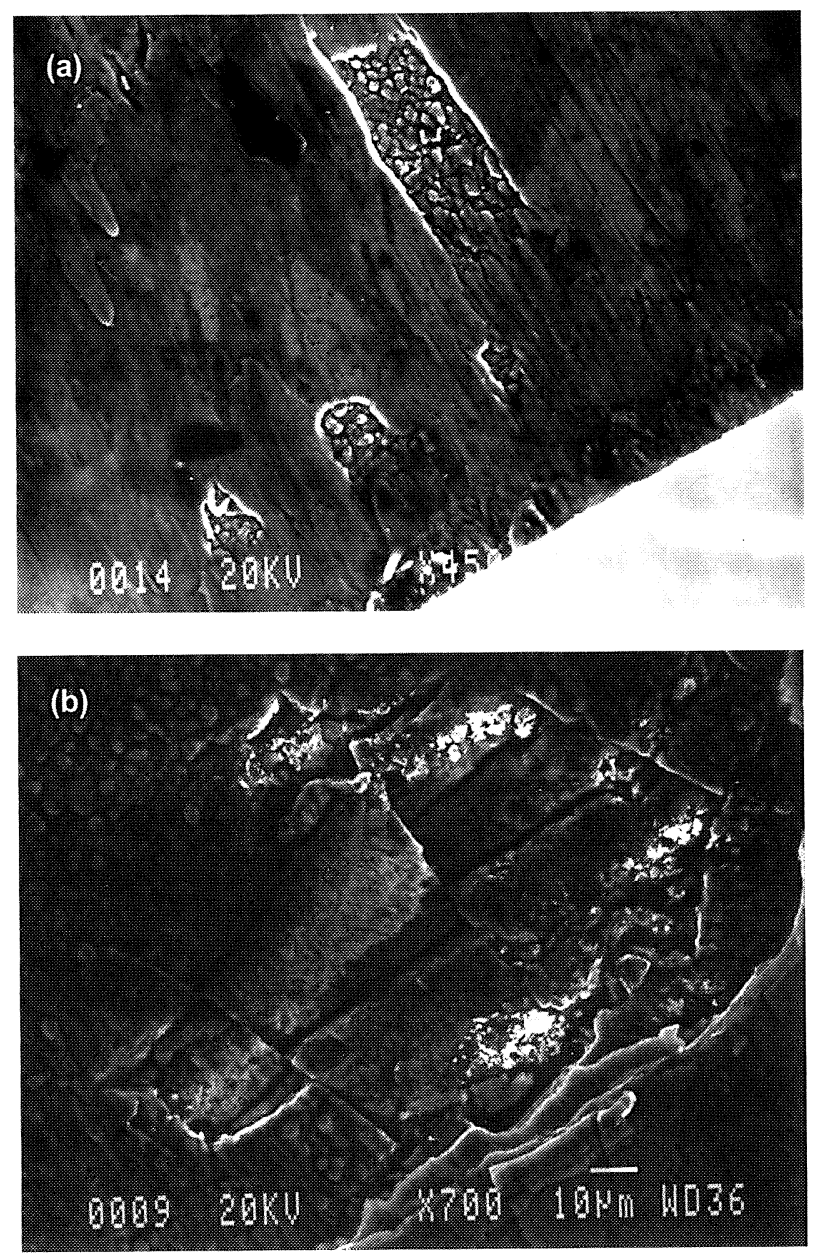

Figura 4. Formación del BUL tras (a) 0,5 y (b) 0,1 s de torneado horizontal de la aleación AA7050. (Herramienta: TiN)

Figure 4. BUL developed after turning AA7050 bars during (a) $0.5 \mathrm{~s}$ and (b) $0.1 \mathrm{~s}$. (Tool: TiN insert).

ayudada por la presión en el momento del corte, dejando libre los intermetálicos, que se irían arrastrados con la viruta. De acuerdo con los comentarios anteriores, este fenómeno se produce en los primeros instantes de mecanizado. Una vez desarrollado el BUL, las condiciones iniciales de corte cambian por el hecho de que el material de la cara de desprendimiento es aluminio, favoreciendo la formación por adhesión mecánica del material mecanizado en la zona del filo, dando origen al BUE ${ }^{[5]}$.

\section{CONCLUSIONES}

Se han identificado mediante SEM los efectos de adhesión para el mecanizado de la aleación AA7050 en un rango de velocidades de corte mo- deradas. Por su parte, el análisis mediante EDS ha permitido distinguir características composicionales diferentes entre capa adherida (BUL) y filo recrecido (BUE) en los procesos estudiados. De acuerdo con los resultados obtenidos, la capa adherida se forma en los primeros instantes de mecanizado y presenta una composición muy cercana a la del aluminio puro. La formación del BUE es posterior y apenas si se observan diferencias entre su espectro EDS y el correspondiente a la aleación original. Estos hechos han permitido establecer una hipótesis preliminar sobre el mecanismo de formación de ambos, a partir de consideraciones sobre la temperatura en la zona de deformación primaria. Según esta hipótesis, la formación del BUL viene precedida de un proceso de fusión incipiente en el que se desprenden los intermetálicos. La existencia del BUL altera la situación inicial de corte y se dan las condiciones para favorecer una adhesión pura de material al filo dando origen al BUE.

\section{Agradecimientos}

Este trabajo ha sido financiado por la Comisión Interministerial de Ciencia y Tecnología (CICYT) proyecto DPI2001-3747, por AIRBUS España, S.L. y por la Junta de Andalucía.

\section{REFERENCIAS}

[1] M.A. Sebastián, J.M. Sánchez, M.S. Carrilero, J.M. González y M. Marcos, Int. J. Manuf. Sci. Prod. 4 (2002) 187-192.

[2] M. SÁnchez-Carrilero, M. Marcos, M. ÁlvarezAlcón y V.M. SÁnCHez-Corbacho, Rev. Metal. Madrid 34 (1998) 27-31.

[3] G. Boothroyd y W.A. KNight, Fundamentals of Machining and Machine Tools, Marcel Dekker Inc., Nueva Cork, EE.UU, 1989.

[4] E.M. TREnT, Metal Cutting, Butterworths, Nueva York, EE.UU, 1989.

[5] M.S. Carrilero, R. Bienvenido, J.M. Sánchez, M. Álvarez, A. GonzÁlez y M. Marcos, Int. J. Mach. Tools Manuf. 42 (2002) 215-220.

[6] J.M. Sánchez, M. Álvarez, M.S. Carrilero, J.M GonzÁlez y M. Marcos, MetalUnivers 14 (2003) 114 117.

[7] M. Bethencourt, F.J. Botana, M.J. Cano y M. Marcos, XV Congr. Nac. Ing. Mec., Libro de Resúmenes, Cádiz, 10 13 diciembre 2002. 